\title{
FREEDOM OF CHOICE: THE LEXIMAX CRITERION IN
} THE INFINITE CASE

\author{
R. Arlegi \\ M. Besada \\ J. Nieto \\ C. Vázquez \\ D.T.2006/08
}




\title{
Freedom of choice: the leximax criterion in the infinite case.
}

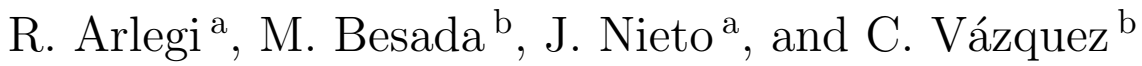 \\ ${ }^{a}$ Universidad Pública de Navarra \\ ${ }^{\mathrm{b}}$ Universidade de Vigo
}

\begin{abstract}
Many recent works have investigated the problem of extending a preference over a set of alternatives to its power set, in an attempt to provide a formal representation of the notion of freedom of choice. In general, results are limited to the finite case, which excludes, for instance, the case of economic environments. This paper deals with the possibility of extending those results to the context where the basic set of alternatives is the $n$-dimensional Euclidean space. We present an extension of the leximax criterion described by Bossert, Pattanaik and Xu (1994) to this more general framework.
\end{abstract}

Key words: preferences, utility, freedom of choice, leximax JEL classification: $\quad$ C60

\section{Introduction}

The aim of this work is to investigate the possibility of extending a preference over a (possibly infinite) set of alternatives to its power set. The motivation for that extension fits into the freedom of choice framework. According to this

* We are grateful to C. Herrero and M. A. Ballester for their help. We appreciate very much suggestions and comments by an anonymous referee and by an editor of this journal. This research has been supported by Spanish Ministry of Education grants SEC2000-0838 (a) and SEC2003-08105 (a) from DGICYT, PGIDIT03PXC30003PN (b) from Xunta de Galicia and BEC2002-3780 (b), from DGICYT.

${ }^{\star \star}$ Please address correspondence to: Jorge Nieto. Department of Economics. Public University of Navarra. Campus Arrosadia. 31600 Pamplona. Navarra. Spain. pho: ++ 948 169365. fax: ++ 34948 169721. e-mail: jnieto@unavarra.es 
approach, the level of well-being enjoyed by an individual is determined by the utility achieved from a given set of alternatives (instrumental value) plus the degree of freedom provided by that opportunity set (intrinsic value).

There have been many relevant works in this field in recent times, starting with the very notion of freedom of choice discussed in Hicks (1959), Buchanan (1986), Dasgupta (1986) and Sen (1988, 1991a, 1991b). The slightly different, though closely related, notion of preference for flexibility first appears in Koopmans (1964) and Kreps (1979) and is developed axiomatically in Arlegi and Nieto (2001).

In Bossert, Pattanaik and $\mathrm{Xu}$ (1994) several rules for comparing subsets of alternatives (opportunities) of a given set are defined and axiomatically characterized; one of these rules is known as the leximax rule. According to this rule, any two sets of alternatives are compared by first looking at the best alternative in each set; if the comparison is not decisive, then the rule considers the second-best alternative, and so the procedure continues until there are no more alternatives to be compared in either or both of the sets. In the first case, the set with more alternatives is declared to be better; in the second one, the two sets are indifferent.

Unfortunately, the leximax rule, as established in Bossert, Pattanaik and $\mathrm{Xu}$ (1994), is defined only when the universe of alternatives is finite. This domain restriction leaves no room for general environments, in which: a) the universe of alternatives is the positive orthant of the $n$-fold cartesian product, and b) individual preferences over this set can be represented by a utility function. In this domain, none of the different rules presented in Bossert, Pattanaik and $\mathrm{Xu}$ (1994) -even the leximax rule- applies. On the other hand, in the classical consumer theory, comparisons between budget sets are made on the sole basis of the indirect utility, which ignores any kind of freedom of choice considerations.

This paper attempts to fill this gap by extending the notion of freedom of choice to the case in which the basic set of alternatives is separable and possibly infinite. In a way, this work follows in the footsteps of Pattanaik and Xu (2000), who study different rankings of compact (and therefore possibly infinite) sets in terms of freedom of choice, based on the "size" of the sets or, alternatively, on the "size of the undominated surface" of the sets. We focus on the leximax rule, and extend it to the case where sets may be infinite, but not necessarily compact. We first establish some independent axioms (some of which match the spirit of those in Bossert, Pattanaik and Xu (1994)), which we apply to the infinite case. We propose a definition of the leximax criterion extended to such a case, and prove a characterization theorem in this environment.

The structure of the paper is as follows: Section 2 presents the notation and 
definitions. Section 3 contains the axioms and some relevant facts deduced from them. In Section 4 we establish the main result and prove the independence of the axioms. Section 5 contains some comments and remarks. The paper concludes with Section 6 (Appendix), where we present the proof of fulfillment of the axioms by the defined criterion.

\section{Notation and definitions}

$\mathbb{N}$ and $\mathbb{R}$ denote, respectively, the set of all natural numbers and the set of all real numbers, respectively, and $\mathbb{R}^{n}$ is the $n$-fold cartesian product of $\mathbb{R}$. Let $X \subset \mathbb{R}^{n}$ be a nonempty set of alternatives. In order to ensure that the axioms used in the characterization are independent, $X$ is assumed to contain at least three elements.

Let $R$ be a complete, reflexive, transitive ordering on $X$, that can be represented by a utility function. The indifference relation associated to $R$ is denoted by $I$, while the strict preference relation is denoted by $P$.

The set of all subsets of $X$, including the empty set, is denoted by $2^{X}$, and $\neg$ denotes the logical negation.

Let $\succ$ be a transitive and asymmetric binary relation defined on $2^{X}$. We write $A \succ B$ to indicate that set $A$ is strictly preferred to set $B$. We define the associated weak preference and indifference relations in the usual way: $\forall A, B \in 2^{X}, A \succeq B$ iff $\neg(B \succ A)$, and $A \sim B$ iff $A \succeq B$ and $B \succeq A$. Note that the negative transitivity of $\succ$ is not assumed. Thus, in this framework, where alternatives may be more complex, the assumptions on $\succ$ display the reduction in the decision maker's capability to rank objects.

We assume $A \succ \emptyset$, for all $A \subset X, A \neq \emptyset$.

We investigate possible preferences over sets of alternatives consistent with a given preference structure defined on the basic alternatives. The formal meaning of consistency will be given by the axioms contained in the next section.

\section{The axioms}

We impose certain properties on the relation between the preference structure on $X$ and the ordering over $2^{X}$. The properties are as follows. 


\section{Dominance (D)}

Let $A, B \subset X$. If, for all $b \in B$, there exists $a \in A$ such that $a P b$, then $A \succeq B$.

\section{Independence (In)}

For all $A, B \subset X$, for all $x, y \in X$, such that $x I y, x \notin A$, and $y \notin B$, then

$$
A \succeq B \Longleftrightarrow A \cup\{x\} \succeq B \cup\{y\} .
$$

\section{Robustness (Rb)}

For all $A, B, C \subset X$, such that $C \cap(A \cup B)=\emptyset$ and verifying that $\forall a \in A$, $\forall b \in B$ and $\forall c \in C, a P c$ and $b R c$, then

$$
A \succ B \quad \Longrightarrow A \succ B \cup C
$$

\section{Indifference (I)}

Let $A, B \subset X$. If there exist two one-to-one functions $\alpha: \mathbb{N} \rightarrow A$ and $\beta: \mathbb{N} \rightarrow$ $B$ such that for all $k \in \mathbb{N}, \alpha(k) I \beta(k), \alpha(k) R a$ for all $a \in A \backslash\{\alpha(1), \ldots, \alpha(k-1)\}$ and $\beta(k) R b$ for all $\beta \in B \backslash\{\beta(1), \ldots, \beta(k-1)\}$, then $A \sim B$.

\section{Interpretation of the axioms}

(D) guarantees that, for any pair of sets $A$ and $B$, such that for all $b \in B$ there exists an $a \in A$ verifying $a R b$, then $A$ is weakly preferred to $B$.

(In) embodies the same spirit as the Independence axiom used by Pattanaik and Xu (1990), but in a weaker form. It states that when two indifferent alternatives are added to, or dropped from sets $A, B$, then the order of preference of the sets will not be reversed. When $y=x$, then the axiom becomes very similar to one used by Pattanaik and Xu (1990), or Bossert, Pattanaik and $\mathrm{Xu}(1994)$.

$(\mathrm{Rb})$ ensures that when a set whose alternatives are worse than any of the alternatives in $A \cup B$ is added to the worst set, $B$, then the ordering between $A$ and $B$ will not change. This is very similar to the "Robustness of Strict Preference" axiom used by Bossert, Pattanaik and Xu (1994) extended to the case in which we add not a single alternative but a set of alternatives. According to their motivation, $(\mathrm{Rb})$ ensures that a lack of indirect utility cannot be compensated by increasing the cardinality of the opportunity set, in the 
sense that this strict preference cannot be undone by adding less attractive alternatives to a set $B$ that is already less desirable than a set $A$. It should be noted that, when trying to consider at once the indirect utility provided by a set and the freedom of choice measured as the number of its alternatives, conflicts between the two values inevitably arise. These conflicts can be resolved by various alternative axioms. In the freedom of choice literature $(\mathrm{Rb})$ is seen simply as a plausible formula to solve them. Further possibilities can be found in Bossert, Pattanaik and Xu (1994).

Axiom (I) is new, and extends the rule to an infinite context. This axiom states that if both sets $A$ and $B$ contain an infinite number of good enough alternatives, which are pairwise indifferent, then $A$ and $B$ must be considered as being indifferent, whatever the remaining alternatives of the sets may be.

In other words, take $A, B \subset X$. Let $A^{\prime}$ and $B^{\prime}$ be two "best" infinite subsets of $A$ and $B$ respectively, whose alternatives are indifferent pairwise, then $A \sim B$. The intuition behind this axiom is that, whenever the comparison between $A^{\prime}$ and $B^{\prime}$ is not decisive, then $A$ and $B$ should be declared indifferent. If all the alternatives in $A^{\prime}$ are indifferent to those in $B^{\prime}$, then it is unnecessary to proceed any further, because if there are infinite indifferences at the highest level, that is enough to make the primitive sets $A$ and $B$ indifferent also.

Axiom (D) guarantees that if $b \in X$ is such that $b P a$ for all $a$ in a certain set $A$, then $\{b\} \succeq A$. The combination of axioms (Rb) and (In) leads to the following results.

Lemma 1 Let $A \subset X, b \in X$. If for all $a \in A, b P a$, then $\{b\} \succ A$.

Proof of lemma 1: Since $\{b\} \succ \emptyset$, then, by (Rb), $\{b\} \succ A$.

Lemma 2 Let $A, B \subset X, A$ finite. If $A \subset B, A \neq B$, then $B \succ A$.

Proof of lemma 2: Since $B \backslash A \succ \emptyset$, then, by (In), $B \succ A$.

As a direct consequence of lemma 1 and (In), we obtain the following property, termed extension (E) in the related literature: for all $x, y \in X, x R y \Longleftrightarrow\{x\} \succeq$ $\{y\}$. This axiom, which is very much a standard in the field, simply states that the preference over alternatives is extended to singletons. This is reasonable when the quality of the alternatives matters, which is not the case in some approaches, where it is only the number of alternatives that matters in the comparison of opportunity sets. Thus, Pattanaik and Xu (1990) studied a case in which the freedom of choice attached to a set of opportunities is measured simply by the number of alternatives. These authors assume that there will be no distinction between sets of alternatives, such as singletons, which offer no 
freedom of choice at all. They then establish that for all $x, y \in X,\{x\} \sim\{y\}$. In this paper it is assumed that the number, as well as the quality of the alternatives, is taken into account when comparing opportunity sets.

\section{A characterization result}

We can now propose an ordering of opportunity sets that satisfies the above axioms, and which is the only one that fulfills such a list of required properties. We call it the leximax ordering on $2^{X}$ and it will be denoted by $\succeq_{L}$. Both the name leximax, and the notation $\succeq_{L}$, have appeared previously in Bossert, Pattanaik and Xu (1994), but unlike them, we use "leximax" and $\succeq_{L}$ to refer to an ordering on $2^{X}$, where $X$ can be infinite.

In order to define the criterion $\succeq_{L}$, a piece of additional notation will be useful. Let $u: X \rightarrow[0,1]$ be such that for all $x, y \in X, u(x) \geq u(y)$ iff $x R y$, that is, $u$ is a utility function.

Let $A \subset X$; we use $u(A)$ to denote the set $\{u(a): a \in A\}$, which implies $u(\emptyset)=\emptyset$. Since $u(A)$ is a bounded set, there exists $\sup u(A)$. If there exists $a \in A$ such that $u(a)=\sup u(A)$, this $a$ will be denoted by $a_{1}$. In this case, we consider the bounded set $u\left(A \backslash\left\{a_{1}\right\}\right)$, then there exists sup $u\left(A \backslash\left\{a_{1}\right\}\right)$; again, if there exists $a \in A \backslash\left\{a_{1}\right\}$ such that $u(a)=\sup u\left(A \backslash\left\{a_{1}\right\}\right)$, this $a$ will be denoted by $a_{2}$, and so on. It is possible, for instance, for there to exist more than one $a \in A$ such that $u(a)=\sup u(A)$, in this event one of them must be chosen to be denoted by $a_{1}$. This choice does not affect the procedure of the criterion.

\section{Definition}

Given a (possibly infinite) set $X$, the leximax ordering on $2^{X}$ is defined as follows:

- $\forall A \neq \emptyset, A \succ_{L} \emptyset \sim_{L} \emptyset$

- $\forall A, B \neq \emptyset$ we consider the following three possibilities:

1) $a_{1}$ exists but not $b_{1}$.

1.1 If $a_{1} P x$, for all $x \in B$, then $A \succ_{L} B$.

1.2 If there exists $x \in B$ such that $x P a_{1}$, then $B \succ_{L} A$.

2) Neither $a_{1}$ nor $b_{1}$ exists.

2.1 If $\sup u(A)>\sup u(B)$, then $A \succ_{L} B$.

2.2 If $\sup u(B)>\sup u(A)$, then $B \succ_{L} A$.

2.3 If $\sup u(A)=\sup u(B)$ then $A \sim_{L} B$. 
3) Both $a_{1}$ and $b_{1}$ exist.

3.1 If $a_{1} P b_{1}$, then $A \succ_{L} B$.

3.2 If $b_{1} P a_{1}$, then $B \succ_{L} A$.

3.3 If $a_{1} I b_{1}$, it could be that:

3.3.a $A=\left\{a_{1}\right\}$ and $B=\left\{b_{1}\right\}$. In which case $A \sim_{L} B$.

3.3.b $A=\left\{a_{1}\right\}$ and $B \neq\left\{b_{1}\right\}$. In which case $B \succ_{L} A$. Analogously, if $A \neq\left\{a_{1}\right\}$ and $B=\left\{b_{1}\right\}$ we declare $A \succ_{L} B$.

3.3.c $A \neq\left\{a_{1}\right\}$ and $B \neq\left\{b_{1}\right\}$. In this case we consider the sets $A \backslash\left\{a_{1}\right\}$ and $B \backslash\left\{b_{1}\right\}$. If $A \backslash\left\{a_{1}\right\}$ and $B \backslash\left\{b_{1}\right\}$ are in one of the cases above (from 1 to 3.3.b), then we conclude $A \succeq_{L} B$ iff $A \backslash\left\{a_{1}\right\} \succeq_{L} B \backslash\left\{b_{1}\right\}$. If not, we repeat the procedure as often as is necessary to find $k \in \mathbb{N}$ such that $a_{i} I b_{i}, i=1, \ldots, k$, and $A \backslash\left\{a_{1}, \ldots, a_{k}\right\}, B \backslash\left\{b_{1}, \ldots, b_{k}\right\}$ are in one of the cases 1 to 3.3.b, concluding $A \succeq_{L} B$ if $A \backslash\left\{a_{1}, \ldots, a_{k}\right\} \succeq_{L} B \backslash\left\{b_{1}, \ldots, b_{k}\right\}$.

The only case in which this algorithm does not come to an end is when, for all $k \in \mathbb{N}$, there exist $a_{k} \in A, b_{k} \in B$ such that, $a_{k} I b_{k}$. In which case, we declare $A \sim_{L} B$.

With respect to this new definition, the following remarks are in order:

(1) For the sake of simplicity, the numbering used above to describe the different possibilities of the definition will be maintained in the proofs of the results.

(2) Let $A$ be a set of alternatives such that there exist $a_{1}, a_{2}, \ldots, a_{k_{o}}$ but not $a_{k_{o}+1}$, and let us identify $A$ with the sequence $U(A)=\left\{u\left(a_{1}\right), u\left(a_{2}\right), \ldots\right.$, $\left., u\left(a_{k_{o}}\right), \sup u\left(A \backslash\left\{a_{1}, \ldots a_{k_{o}}\right\}\right), 0, \ldots\right\}$. Note, then, that in order to relate a pair of sets, $A$ and $B$, we are in fact lexicographically comparing $U(A)$ and $U(B)$, with a restriction in a very particular case: if there exist $a_{k}$ and $b_{k}$, for $k=1, \ldots k_{o}$ such that $a_{k} I b_{k}$; there exists $a_{k_{o}+1}$; there does not exist $b_{k_{o}+1}$; and $u\left(a_{k_{o}+1}\right)=\sup u\left(B \backslash\left\{b_{1}, \ldots, b_{k_{o}}\right\}\right)$, then $A \succ_{L} B$, instead of checking the subsequent elements of both $U(A)$ and $U(B)$. Thus, the leximax ordering we propose gives priority to the value of the maximal of $U(A)$ against the supremum, when the latter does not belong to the set.

(3) The above definition is invariant with respect to any utility representation. The utility representation is used only in case 2) of the definition, but all its subcases 2.1, 2.2 and 2.3 could be rewritten in terms of the underlying preferences.

(4) The leximax rule is indeed an ordering. Its definition establishes the completeness of the ordering. In order to prove the reflexivity of the binary relation, we consider three cases for any given set $A$ :

(a) There exist $a_{1}, \ldots, a_{k_{o}-1}$ but not $a_{k_{o}}$, with $k_{o} \in \mathbb{N}$. From 3.3 , to compare $A$ with itself, we must consider $\sup u\left(A \backslash\left\{a_{1}, \ldots, a_{k_{o}-1}\right\}\right)$ and $\sup u\left(A \backslash\left\{a_{1}, \ldots, a_{k_{o}-1}\right\}\right)$. Then, by 2.3 in the definition, $A \sim_{L} A$.

(b) There exist $a_{k} \in A$, for all $k \in \mathbb{N}$, thus by 3.3 in the definition 
$A \sim_{L} A$.

(c) $A$ is a finite set. Thus, by $3.3, A \sim_{L} A$.

$\succeq_{L}$ is also transitive: assume $A, B$ and $C$, such that $A \succeq_{L} B$ and $B \succeq_{L} C$. Then eight cases are considered:

(a) $a_{1}, b_{1}$ and $c_{1}$ do not exist. Then, $\sup u(A) \geq \sup u(B) \geq \sup u(C)$ and, by 2.2 and $2.3, A \succeq_{L} C$.

(b) There exists $a_{1}$ but neither $b_{1}$ nor $c_{1}$ exists. Then, $a_{1} P b$, for any $b \in B$ and $\sup u(B) \geq \sup u(C)$. Therefore $a_{1} P c$, for all $c \in C$. Thus, by $1.1, A \succ_{L} C$.

(c) There exists $b_{1}$ but neither $a_{1}$ nor $c_{1}$ exists. This means that there exists $\bar{a} \in A$, such that $\bar{a} P b_{1}$ and $b_{1} P c$, for any $c \in C$. Then

$$
\sup u(A) \geq u(\bar{a})>u\left(b_{1}\right) \geq u(c), \text { for all } c \in C,
$$

therefore, $\sup u(A)>\sup u(C)$, and, by $2.2, A \succ_{L} C$.

(d) Neither $a_{1}$ nor $b_{1}$ exists, but $c_{1}$ does. We infer that $\sup u(A) \geq$ $\sup u(B)$ and also the existence of $\bar{b} \in B$, such that $\bar{b} P c_{1}$. Thus, there exists $\bar{a} \in A$ verifying $\bar{a} R \bar{b}$. By the transitivity of $P, \bar{a} P c_{1}$, and $A \succ_{L} C$ is held.

(e) Both $a_{1}$ and $b_{1}$ exist but $c_{1}$ does not. We have $a_{1} R b_{1}$ and $b_{1} P c$ for all $c \in C$, then $a_{1} P c$ for all $c \in C$, therefore $A \succ_{L} C$.

(f) Both $a_{1}$ and $c_{1}$ exist, but $b_{1}$ does not. For all $b \in B, a_{1} P b$ is obtained and, also the existence of $\bar{b} \in B$, such that $\bar{b} P c_{1}$. Therefore $a_{1} P c_{1}$ and we have $A \succ_{L} C$.

(g) $a_{1}$ does not exist, but $b_{1}$ and $c_{1}$ do. It follows that there exists $\bar{a} \in A$ such that $\bar{a} P b_{1}$ and again $b_{1} R c_{1}$ is verified. Taking this into account, we have $\bar{a} P c_{1}$, therefore $A \succ_{L} C$.

(h) There exist $a_{1}, b_{1}$ and $c_{1}$. In this case $a_{1} R b_{1} R c_{1}$. We distinguish two subcases.

(i) If $a_{1} P b_{1}$ or $b_{1} P c_{1}$, clearly $A \succ_{L} C$.

(ii) If $a_{1} I b_{1} I c_{1}$, we repeat the above process with $a_{2}, b_{2}$ and $c_{2}$ in place of $a_{1}, b_{1}$ and $c_{1}$, to obtain $A \succeq_{L} C$. In the event of $a_{k} \sim c_{k}$ for all $k \in \mathbb{N}, A \sim_{L} C$ is held. Finally, if $A, B$ or $C$ are finite, it is easy to show that $A \succeq_{L} C$.

The leximax ordering, such as defined in Bossert, Pattanaik and Xu (1994) for finite sets, (we will call it the "finite-leximax" in order to distinguish between the two versions) combines both the intrinsic and the instrumental value of freedom of choice. Indeed that was the authors' main motivation for proposing such a rule. In other words, their proposal accommodates not only the role of preferences, but also the number of alternatives. On the one hand, the role played by the preferences is hightened by the fact that the finite-leximax coincides with the Indirect Utility Criterion whenever there are no ties between 
the two respective best alternatives for a pair of sets. However, the "numberof-alternatives-factor" resides in the fact that, in the event of a tie, instead of declaring both sets indifferent (as the Indirect Utility Criterion would) the rule considers further alternatives. Roughly speaking, the finite-leximax can be understood as a successive replication of the Indirect Utility Criterion until ties between best elements disappear, a replication in which we successively eliminate alternatives as long as they are tied. Therefore, the finite-leximax rule only declares two sets to be indifferent when, after all possible replications, ties cannot be broken.

The extension of the finite-leximax proposed for infinite sets maintains all these features. In the infinite case, however, the idea of a tie between maximal elements is more complex. We therefore identify two possible tie situations: a) as in the finite case, if in a pair of sets both best elements coincide and belong to the set, or b) a pair of sets have no best element but their respective suprema coincide. Having established what we mean by a "tie", the extension we propose retains the essence of the finite version. Likewise, whenever there are no ties Indirect Utility has priority. On the other hand, whenever a tie arises, as in the finite-leximax, we eliminate the tied elements and reapply the Indirect Utility Criterion. Our extension of the leximax, like the finite-leximax, declares two sets to be indifferent only after successive iterations have failed to break successive ties. But in our context there are again two circumstances in which it is impossible to break the tie: 1) when both sets are finite, and all the elements are indifferent two by two, or 2) when both sets are infinite and successive application of the algorithm implies applying it infinite times.

Note also that our extended leximax ignores the worst alternatives in an infinite set $A$ where there exists $k_{o} \in \mathbb{N}$ such that there exist $a_{1}, \ldots, a_{k_{o}}$ but not $a_{k_{o}+1}$. We think that, given the availability of an infinite number of alternatives "close to $a_{k_{o}+1}$ ", less desirable alternatives lose their relevance.

The leximax rule satisfies (D), (In) (Rb) and (I). The main result of our work is the proof that this is the only rule that satisfies these axioms.

Lemma 3 The leximax rule defined $\succeq_{L}$ satisfies Dominance.

Lemma 4 The leximax rule $\succeq_{L}$ satisfies Independence.

Lemma 5 The leximax rule $\succeq_{L}$ satisfies Robustness.

Lemma 6 The leximax rule $\succeq_{L}$ satisfies Indifference.

(Proofs of Lemmata 3 to 6 are provided in the Appendix)

Theorem 7 Let $\succeq$ be an ordering on $2^{X}$. 
$\succeq$ satisfies (D), (In), (Rb) and (I) if and only if $\succeq_{=} \succeq_{L}$.

\section{Proof of Theorem 7}

For notational convenience, throughout the proofs given in the paper, for any pair of sets $A, B$ we assume that the first step of the rule (whether or not $a_{1}$ and $b_{1}$ exist) is the decisive one. Note that, if there exists a finite number of pairs of initial elements that are indifferent two by two, and subsequently this situation is not repeated, then the reasoning is not affected by eliminating the initial pairwise indifferent elements.

As has been proved by lemmas 3, 4, 5 and $6, \succeq_{L}$ satisfies (D), (In), (Rb) and (I). Therefore we have only to prove the sufficient part of the theorem: if $\succeq$ is an ordering on $2^{X}$ verifying $(\mathrm{D}),(\mathrm{In}),(\mathrm{Rb})$ and $(\mathrm{I})$, then $\succeq_{=} \succeq_{L}$.

Given that for all nonempty set $A \in 2^{X}, A \succ_{L} \emptyset \sim_{L} \emptyset$ directly follows from the assumptions on $\succeq$, we shall concentrate on the case in which $A, B \neq \emptyset$.

We will start by supposing that $A \sim_{L} B$ and then prove that $A \sim B$. There are two possible cases:

Case 2.3 Neither $a_{1}$ nor $b_{1}$ exists, and $\sup u(A)=\sup u(B)$. Then, for any $b \in B$, there exists $a \in A$ such that $a P b$ and, by (D), we have that $A \succeq B$. By analogy, for every $a \in A$, there exists $b \in B$ such that $b P a$, and again by (D), we arrive at $B \succeq A$. From both relations we have $A \sim B$.

Case 3.3 If $A=\left\{a_{1}\right\}, B=\left\{b_{1}\right\}$, by (In), $A \sim B$. If $A \neq\left\{a_{1}\right\}, B \neq\left\{b_{1}\right\}$ there are two possibilities: Either both $A$ and $B$ are finite or neither $A$ nor $B$ is finite. In the first case, by (In) $A \sim B$. In the second case, by (I) $A \sim B$

We now suppose that $A \succ_{L} B$ and we prove that this implies $A \succ B$. The possible cases are:

Case 1.1 There exists $a_{1} \in A$, there does not exist $b_{1} \in B$, and $a_{1} P x$, for all $x \in B$. By lemma $1\left\{a_{1}\right\} \succ B$. If $A \neq\left\{a_{1}\right\}$, by lemma 2 we obtain $A \succ\left\{a_{1}\right\}$. In any case, $A \succeq\left\{a_{1}\right\}$, thus $A \succ B$.

Case 1.2 There does not exist $a_{1} \in A$, there exists $b_{1} \in B$ and there exists $a \in A$ such that $a P b_{1}$. By lemma $1\{a\} \succ B$. By lemma 2, $A \succ\{a\}$, then $A \succ B$.

Case 2.1 There do not exist $a_{1} \in A, b_{1} \in B$ and $\sup u(A)>\sup u(B)$. Then there exists $a \in A$ such that $a P b$ for all $b \in B$ and then, by lemma 1, we have $\{a\} \succ B$, and by lemma $2 A \succ B$. 
Case 3.1 There exist $a_{1} \in A, b_{1} \in B$ and $a_{1} P b_{1}$. If $A \neq\left\{a_{1}\right\}$, by lemma 2, $A \succ\left\{a_{1}\right\}$. In any case, $A \succeq\left\{a_{1}\right\}$. On the other hand, by lemma 1 , we have $\left\{a_{1}\right\} \succ B$, then $A \succ B$.

Case 3.3 There exist $a_{1} \in A, b_{1} \in B$ such that $a_{1} I b_{1}, A \neq\left\{a_{1}\right\}$ and $B=\left\{b_{1}\right\}$. Since $\left\{a_{1}\right\} \subset A$, there exists $a_{2} \neq a_{1}$ such that $a_{2} \in A$. Also, since $\left\{a_{2}\right\} \succ \emptyset$, by (In) $\left\{a_{1}, a_{2}\right\} \succ\left\{b_{1}\right\}$. If $A=\left\{a_{1}, a_{2}\right\}$ we are done. If $\left\{a_{1}, a_{2}\right\} \subset A$, by Lemma $2 A \succ\left\{a_{1}, a_{2}\right\}$, and by transitivity $A \succ B$.

Remark: Notice that in the proof, we did not directly appeal to axioms (Rb) and (In). The reason is that, together, they imply lemmas 1 and 2, which are repeatedly used in the proof. Therefore an alternative presentation of Theorem 7 could be formulated by dropping axioms ( $\mathrm{Rb}$ ) and (In), and replacing them with lemmas 1 and 2 .

The next result proves the independence of the axioms used in Theorem 7 .

Theorem 8 Axioms (D), (In), (Rb) and (I) are independent.

\section{Proof of Theorem 8}

(1) Consider $X=\mathbb{R}$ where all alternatives are indifferent. Let $A \subset \mathbb{R}$, and let $u(A)=\sharp A$ if $A$ is a finite subset, $u(A)=\aleph_{o}$ if $A$ is a infinite countable subset and $u(A)=\aleph_{1}$ if $A$ is a uncountable subset. The preference relation on $2^{X}$ defined by $u$ satisfies $(\mathrm{D}),(\mathrm{In})$ and $(\mathrm{Rb})$ but not (I).

(2) Consider a finite set $X$ with at least three elements where a preference relation $R$ is defined. Let $A, B \subset X$, we define $A \succeq B$ iff $a_{1} R b_{1}$, with $a_{1}$ and $b_{1}$ the best alternatives on $A$ and $B$ respectively. This preference relation on $2^{X}$ satisfies $(\mathrm{D}),(\mathrm{I})$ and $(\mathrm{Rb})$ but not $(\mathrm{In})$.

(3) Consider a finite set $X$ with at least three elements where a preference relation $R$ is defined. The lexicographic maximin relation defined on $2^{X}$ (see Pattanaik and Peleg (1990)) satisfies (In), (I) and (Rb) but not (D).

(4) Consider $X=\{x, y, z\}$ where $x P y P z$. We define on $2^{X}$ the linear preference relation $\{x, y, z\} \succ\{x, y\} \succ\{x, z\} \succ\{y, z\} \sim\{x\} \succ\{y\} \succ$ $\{z\} \succ \emptyset$. This preference relation on $2^{X}$ satisfies (In), (I) and (D) but not $(\mathrm{Rb})$.

\section{Discussion and conclusions}

(1) Note that we do not assume transitivity of the indifference, which in our context might be too restrictive. Since $\sim_{L}$ is transitive, the transitivity 
of $\sim$ is a consequence of the axioms and the assumptions of asymmetry and transitivity of $\succ$.

(2) The result presented in the theorem can be seen as an extension of the leximax rule defined by Bossert, Pattanaik and Xu (1994) to a general domain where the universal set of alternatives is the euclidean space, and the decision maker's preferences over this domain are representable on the real line. Although the leximax procedure is usually defined for the finite case, this article presents an extension to suit the continuous case. In this extension we use the fact that the sets to be compared are bounded, and thus have suprema. The comparison of sets applies the lexicographic procedure to a finite list of (possibly infinite) bounded sets.

(3) The results are equally valid if we consider the domain of preferences and axioms to be any arbitrary collection of subsets of $X$ satisfying certain conditions. In particular, let $\chi$ denote any family of subsets of $X$ satisfying the following:

(a) All singletons of $X$ belong to $\chi$.

(b) $\chi$ is comprehensive: If $A \in \chi$, then, for all $B \subset A, B \in \chi$.

(c) $\chi$ is closed by the union: For all $A, B \in \chi, A \cup B \in \chi$.

The three properties above ensure the axioms to be well-defined and the proofs to go through. Therefore, our characterization still holds for meaningful specifications of $\chi$ such as the family of all finite subsets of $X .^{1}$

(4) As said before, the leximax criterion characterized in the previous sections reproduces the leximax rule defined for finite sets in Bossert, Pattanaik and Xu (1994). Therefore, the set axioms used by Bossert, Pattanaik and $\mathrm{Xu}$ (1994) should be equivalent to the those used in our work when restricted to a finite domain. Such equivalence, however, can be proved only after some minor rearrangements. The reason is that, even in a finite framework, there are slight differences between the two models: Bossert, Pattanaik and $\mathrm{Xu}$ (1994) start, for the sake of simplicity, from a linear ordering $R$ over $X$ (complete, transitive and antisymmetric), while our primitive relation $R$ is assumed to be an ordering (complete, transitive and reflexive). A second difference is the treatment of the empty set, which is outside the domain in Bossert, Pattanaik and Xu's case. If we considered our $R$ to be a linear ordering on $X$, and if we extended Bossert, Pattanaik and Xu's domain to include the empty set as the worst set, then the equivalence between the two referred groups of axioms can be proved in the finite case. The proof is available from the authors upon request.

1 The family of compact sets, which is also meaningful in economic contexts, is not comprehensive. Comprehensiveness is only required for the proof of Lemma 2 . Therefore, any of the results in the article could be proved for the family of compact sets with axioms (D), (In), (Rb) and (I) plus the property stated by Lemma 2, which, for this particular case, is independent of the previous four. 
(5) Note that, in many cases, the leximax rule characterized in this paper serves to show the intrinsic value of freedom of choice, while the standard indirect utility rule does not. For example, let $A$ be a classical budget set, with $a_{1}$ as its best alternative according to relation $R$ (see figure 1 ). Following the standard criterion of consumer theory, $A \sim\left\{a_{1}\right\}$, while according to the leximax rule, $A \succ\left\{a_{1}\right\}$.

(6) Ballester, de Miguel and Nieto (2004) have defined and characterized the extension of the classical Indirect Utility Criterion to a similar domain of infinite environments. One of the key axioms used in this result (Useless Freedom of Choice) states that, for all $x, y \in X, x P y$ implies $\{x, y\} \sim\{x\}$. This axiom is violated by the leximax rule. On the other hand, the leximax also violates one of the key axioms of Pattanaik and Xu's Cardinalistic Criterion, namely, Indifference Between No-Choice Situations, which requires $\{x\} \sim\{y\}$, for all $x, y \in X$.

The above reflects the fact that the leximax criterion is supposed to represent a compromise between those extreme positions and shares some (but not all) of their features. Since the aim is to achieve a compromise, properties that lean heavily towards one of the values (intrinsic or instrumental) must be rejected.

(7) Pattanaik and $\mathrm{Xu}$ (2000) propose different criteria for comparing compact sets in terms of freedom of choice. Their criteria are based on the size of the sets, measured by their volume (or area in the case of budget sets). For example, let $A, B$ be two budget sets such that $B \subset A$ and their best alternatives coincide, $a_{1}=b_{1}$ (see figure 2). According to Pattanaik and Xu's approach, since $A$ 's area is bigger than $B$ 's, $A$ should be declared better than $B$.

However, according to the leximax rule, in the situation depicted in figure $2, A \sim_{L} B$. Note that this seems to contradict the idea of freedom of choice, as set $A$ is bigger than set $B$. However, it can also be interpreted from another point of view that maintains the spirit of freedom of choice. In fact the reasons for $A$ being indifferent to $B$ are, first, that there are the same number of alternatives in $A$ as in $B$, and, second, that these alternatives are equally desirable, that is, for each alternative in $A$, different from $a_{1}$, there is at least one alternative in $B$ that is just as desirable, and vice-versa.

The example above illustrates the different treatment given here to the notion of freedom of choice with respect to Pattanaik and Xu (2000). As we have already pointed out, Pattanaik and Xu's approach is based on the size of the set, measured by its area in the case of budget sets, while our treatment has more to do with the number of alternatives the set contains. In the finite case the number of alternatives provides a proper measure of the size of the set, so the above mentioned distinction becomes vacuous. However, when considering infinite sets, this distinction acquires full relevance: A set $A$ might be bigger in area than another set $B$ (as in figure 2), while having the same (infinite) number of alternatives. 


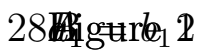

\section{Appendix}

\section{Proof of lemma 3}

Suppose that for all $b \in B$, there exists $a \in A$ such that $a P b$; we have to prove that $A \succeq_{L} B$. The possibilities, which depend on the existence of suprema, are as follows:

1) $a_{1}$ exists but $b_{1}$ does not. Then $a_{1} P b$ for all $b \in B$, and, by using 1.1 we have $A \succ_{L} B$.

1') $b_{1}$ exists but $a_{1}$ does not. The hypothesis guarantees that there exists $a \in A$ such that $a P b_{1}$, then, by $1.2, A \succ_{L} B$.

2) Neither $a_{1}$ nor $b_{1}$ exists. The hypothesis implies $\sup u(A) \geq \sup u(B)$ and, by 2.1 and 2.3 , we obtain $A \succeq_{L} B$.

3) Both $a_{1}$ and $b_{1}$ exist. Then necessarily $a_{1} P b_{1}$, and case 3.1 implies that $A \succ_{L} B$

\section{Proof of lemma 4}

Let $x, y \in X$ such that $x I y, x \notin A$ and $y \notin B$. We will prove that $A \succeq_{L}$ $B \Longleftrightarrow A \cup\{x\} \succeq_{L} B \cup\{y\}$.

" $\Longrightarrow$ "The assumption $A \succeq_{L} B$ could occur in six different cases.

Case $1.1 a_{1}$ exists but $b_{1}$ does not, and $a_{1} P b$, for all $b \in B$. It could be that: a) $x R a_{1}$. In this situation there exist $\overline{a_{1}}=x$ and $\overline{b_{1}}=y$, such that

$$
u\left(\overline{a_{1}}\right)=\sup u(A \cup\{x\})=u\left(\overline{b_{1}}\right)=\sup u(B \cup\{y\}) .
$$

Taking into account 3.3, in order to compare $A \cup\{x\}$ and $B \cup\{y\}$, we only need to compare $A$ and $B$, therefore $A \cup\{x\} \succeq_{L} B \cup\{y\}$.

b) $a_{1} P x$. Under these conditions $u\left(a_{1}\right)=\sup u(A \cup\{x\})$. Moreover, $a_{1} P x$, $x I y$ and $a_{1} P b$, for all $b \in B$, then $a_{1} P b$, for all $b \in B \cup\{y\}$, and by 1.1, $A \cup\{x\} \succ_{L} B \cup\{y\}$.

Case $1.2 b_{1}$ exists but $a_{1}$ does not, and there exists $a_{o} \in A$ such that $a_{o} P b_{1}$. If $x R a$, for all $a \in A$ the reasoning is the same as for case 1.1.-a), if $\neg(x R a)$, there exists $a^{\prime} \in A$ such that $a^{\prime} P x$ and, in addition, the supremum is not reached in $A \cup\{x\}$. Thus, denoting by $\bar{b}_{1}=\max _{R}\left\{y, b_{1}\right\}$, if $\bar{a}=\max _{R}\left\{a, a^{\prime}\right\}$, then $u\left(\bar{b}_{1}\right)=\sup u(B \cup\{y\})$ and $\bar{a} P \bar{b}_{1}$, and, by 1.2, $A \cup\{x\} \succ_{L} B \cup\{y\}$. 
Case 2.1 Neither $a_{1}$ nor $b_{1}$ exists, and $\sup u(A)>\sup u(B)$. Note that $\sup u(A \cup$ $\{x\})=\max \{\sup u(A), u(x)\}$, therefore, if $u(x) \geq \sup u(A)$, by using 3.3 the alternatives $x$ and $y$ must be removed from the sets we intend to compare, thus, by 2.1, $A \cup\{x\} \succeq_{L} B \cup\{y\}$.

On the other hand, if $\sup u(A)>u(x)$, the supremum is not reached in $A \cup\{x\}$ and $\sup u(A \cup\{x\})=\sup u(A)$. In the additional event that $u(x) \geq \sup u(B)$, the supremum is reached in $B \cup\{y\}$ (at $y)$ but $\sup u(A \cup\{x\})>u(x)=$ $u(y)=\sup u(B \cup\{y\})$, and, by 1.2, $A \cup\{x\} \succ_{L} B \cup\{y\}$. Otherwise, if $u(x)<\sup u(B)$, the supremum is not reached either in $A \cup\{x\}$ or in $B \cup\{y\}$ and $\sup u(A \cup\{x\})=\sup u(A)>\sup u(B)=\sup B \cup\{y\}$, which by 2.1 implies that $A \cup\{x\} \succ_{L} B \cup\{y\}$.

Case 2.3 Neither $a_{1}$ nor $b_{1}$ exists, and $\sup u(A)=\sup u(B)$. If $u(x) \geq \sup u(A)$, $x$ and $y$ must be removed from $A \cup\{x\}$ and $B \cup\{y\}$, respectively and, by 2.3, $A \cup\{x\} \sim_{L} B \cup\{y\}$ is obtained. Otherwise, if $u(x)<\sup u(A)$, then

$$
\sup u(A \cup\{x\})=\sup u(A)=\sup u(B)=\sup u(B \cup\{y\}),
$$

thus $A \cup\{x\} \sim_{L} B \cup\{y\}$.

Case 3.1 There exist $a_{1}$ and $b_{1}$ with $a_{1} P b_{1}$. In the additional event that $x R a_{1}$, by 3.3, we must drop $x$ and $y$ and compare sets $A$ and $B$, thus $A \cup\{x\} \succ_{L}$ $B \cup\{y\}$ is obtained. Otherwise, if $a_{1} P x$, the supremum of $u(A \cup\{x\})$ and $u(B \cup\{y\})$ is reached at $a_{1}$ and $\bar{b}_{1}=\sup \left\{y, b_{1}\right\}$ respectively. In both cases $a_{1} P \bar{b}_{1}$ and 3.1 guarantees $A \cup\{x\} \succ_{L} B \cup\{y\}$.

Case 3.3 There exist $a_{1}$ and $b_{1}$ with $a_{1} I b_{1}$. If there exist $a_{k} \in A$ and $b_{k} \in B$, and $a_{k} I b_{k}$, for all $k \in \mathbb{N}$, there could be:

a) $x P a_{k}$, for all $k \in \mathbb{N}$. Noting that $a_{o}=x$ and $b_{o}=y$, and by using 3.3 with the sequences $\left\{a_{k}\right\}$ and $\left\{b_{k}\right\}, k \in \mathbb{N} \cup\{0\}, A \cup\{x\} \sim_{L} B \cup\{y\}$ is obtained.

b) There exists $k_{o} \in \mathbb{N}$ such that $a_{k_{o}-1} R x R a_{k_{o}}$. Using 3.3 with the sequences $\left\{a_{1}, \ldots, a_{k_{o}-1}, x, a_{k_{o}}, \ldots\right\}$ and $\left\{b_{1}, \ldots, b_{k_{o}-1}, y, b_{k_{o}}, \ldots\right\}, A \cup\{x\} \sim_{L} B \cup\{y\}$ is also verified.

c) $a_{k} P x$, for all $k \in \mathbb{N} . A \cup\{x\} \sim_{L} B \cup\{y\}$ is again obtained, by using 3.3 with the original sequences $\left\{a_{k}\right\}$ and $\left\{b_{k}\right\}$.

If $B=\left\{b_{1}\right\}$, there could be

$x R a_{1}$ : Noting again that $a_{o}=x$ and $b_{o}=y$, by using 3.3 , we claim that $A \cup\{x\} \sim_{L} B \cup\{y\}$.

or $a_{1} P x$ : Then, by 3.3, $A \cup\{x\} \sim_{L} B \cup\{y\}$. 
" $\Longleftarrow "$ Throughout this part of the proof we denote the best elements in $A \cup\{x\}$ and $B \cup\{y\}$ by $a_{1}$ and $b_{1}$ respectively. The assumption $A \cup\{x\} \succeq_{L}$ $B \cup\{y\}$ could occur in five cases.

Case $1.1 a_{1}$ exists, the supremum is not reached in $B \cup\{y\}$, and $a_{1} P b$, for all $b \in B \cup\{y\}$. Then, since $x I y, a_{1} \neq x$. Thus $u\left(a_{1}\right)=\sup u(A)$ and $a_{1} P b$, for all $b \in B$. By 1.1, $A \succ_{L} B$.

Case $1.2 b_{1}$ exists but $a_{1}$ does not, and there exists $a \in A \cup\{x\}$ such that $a P b_{1}$. Note that $x=a$ would imply that $x P b_{1} R y$, therefore $a \neq x$. Thus, if the supremum is reached in $B$ (at element $\overline{b_{1}}$ ), $a P b_{1} P \overline{b_{1}}$, and then $A \succ_{L} B$. Otherwise, if the supremum is not reached in $B$, then $a P b_{1}=y R b$, for all $b \in B$. In this situation, the supremum is not reached in either $A$ or $B$, and there exists $a \in A$ verifying that $a P b$ for all $b \in B$. Then $\sup u(A)>\sup u(B)$ and, by $2.1, A \succ_{L} B$.

Case 2 The supremum is not reached in either $A \cup\{x\}$ or $B \cup\{y\}$, and $\sup u(A \cup$ $\{x\}) \geq \sup u(B \cup\{y\})$. Consequently the supremum is not reached in either $A$ or $B, \sup u(A \cup\{x\})=\sup u(A)$ and $\sup u(B \cup\{y\})=\sup u(B)$. Then $A \succeq_{L} B$.

Case 3.1 Both $a_{1}, b_{1}$ exist, and $a_{1} P b_{1}$. It is verified that $x \neq a_{1}$, then $u\left(a_{1}\right)=$ $\sup u(A)$ and $a_{1} P b$ for all $b \in B$. In the event that the supremum is reached at $\overline{b_{1}}$ in $B$, we have $a_{1} P \overline{b_{1}}$ and, by $3.1, A \succ_{L} B$. Otherwise, if the supremum is not reached in $B$, we have $a_{1} P b$ for all $b \in B$ and, by 1.1, $A \succ_{L} B$.

Case 3.3 If there exist $a_{k} \in A \cup\{x\}$ and $b_{k} \in B \cup\{y\}$, for all $k \in \mathbb{N}$, and $a_{k} I b_{k}$. If $a_{k} \neq x$ and $b_{k} \neq y$ for all $k \in \mathbb{R}$, then, by using 3.3, with the same sequences, $A \succ_{L} B$ is obtained. If there exists $k_{1} \in \mathbb{N}$ such that $a_{k_{1}}=x$, we have $y I x=a_{k_{1}} I b_{k_{1}} R b_{k}$, for all $k \geq k_{1}$. In the additional event that there exists $k_{2} \neq k_{1}$ such that $a_{k_{1}}=x$ and $b_{k_{2}}=y$, we remove the element $k_{1}$ from both sequences and change the element $k_{2}$ from the $b_{k}$-sequence for $b_{k_{1}}$ to obtain a new pair of sequences of elements of $A$ and $B$. Then, under the conditions of 3.3, $A \succ_{L} B$. Otherwise, if $b_{k} \neq y$, for all $k \in \mathbb{N}$, we only remove element $k_{1}$ from both sequences to obtain a pair of sequences on $A$ and $B$ respectively under the conditions of case 3.3, then $A \succ_{L} B$ is obtained too.

If $A$ and/or $B$ are finite sets, by similar steps it can be established that $A \succ_{L}$ $B$.

Proof of lemma 5 Let $A, B, C \subset X$, such that $C \cap(A \cup B)=\emptyset$ and verifying that $\forall a \in A, \forall b \in B$ and $\forall c \in C, a P c$ and $b R c$. Suppose that $A \succ_{L} B$, there are five possibilities:

Case $1.1 a_{1}$ exists but $b_{1}$ does not, and $a_{1} P b$ for all $b \in B$. Since $b R c \forall b \in B$ 
and $\forall c \in C$, the supremum in $B \cup C$ is not reached. Moreover, $a_{1} P c$ for all $c \in C$, then $a_{1} P x$, for all $x \in B \cup C$, then by $1.1, A \succ_{L} B \cup C$.

Case $1.2 b_{1}$ exists, $a_{1}$ does not, and there exists $a \in A$ such that $a P b_{1}$. In addition we have $b_{1} R c$, for all $c \in C$, then the supremum of $B \cup C$ is reached at $b_{1}$, and by $1.2, A \succ_{L} B \cup C$.

Case 2.1 Neither $a_{1}$ nor $b_{1}$ exists, and $\sup u(A)>\sup u(B)$. In this case the supremum of $B \cup C$ is again not reached and, in addition, $\sup u(B)=\sup u(B \cup$ $C)$. Thus, by 2.1, $A \succ_{L} B \cup C$.

Case 3.1 There exist $a_{1}$ and $b_{1}$ verifying that $a_{1} P b_{1}$. Clearly, the supremum of $B \cup C$ is reached at $b_{1}$, then, by $3.1, A \succ_{L} B \cup C$.

Case 3.3 There exist $a_{1}$ and $b_{1}$, verifying that $a_{1} I b_{1}, A \neq\left\{a_{1}\right\}$ and $B=\left\{b_{1}\right\}$. We must compare $A \backslash\left\{a_{1}\right\}$ and $C$. Since $a P c$, for all $a \in A$ and $c \in C, A \succ_{L} C$ is held, whether $a_{2}$ and $c_{1}$ exist or not. Then $A \succ_{L} B \cup C$.

Proof of lemma 6 Let $A, B \subset X$, and let $\left\{a_{k}\right\} \subset A$ and $\left\{b_{k}\right\} \subset B$ be sequences verifying the conditions of the Indifference axiom, in view of case 3.3, we have $A \sim_{L} B$.

\section{References}

Arlegi R., Nieto J., 2001. Ranking opportunity sets: an approach based on the preference for Flexibility. Social Choice and Welfare 18(1), 23-36.

Ballester M., de Miguel J., Nieto J., 2004. Set comparisons in a general domain: the indirect utility criterion. Mathematical Social Sciences, in press.

Bossert W, Pattanaik P.K., Xu Y., 1994. Ranking opportunity sets: An axiomatic approach. Journal of Economic Theory 63, 326-345.

Buchanan J., 1986. Liberty, Market and the State. Wheatsheaf Books, Brighton.

Dasgupta P., 1986. Positive Freedom, markets and the welfare state. Oxford Review of Economic Policy 2, 25-36.

Hicks J., 1959. A manifesto. Reprinted in J. Hicks, Wealth and Welfare. Basil Blackwell, Oxford.

Koopmans T.C., 1964. On the flexibility of future preferences. In Shelley MW and Bryan JL (eds.) Human Judgments and Optimality, Wiley. New York.

Kreps D.M., 1979. A representation theorem for Preference for Flexibility. Econometrica 47, 565-577

Pattanaik P.K., Peleg P., 1984. An axiomatic characterization of the lexicographic maximin extension of an ordering over a set to the power set. Social Choice and Welfare 1, 113-122. 
Pattanaik P.K., Xu Y., 1990. On Ranking Opportunity sets in Terms of Freedom of Choice. Recherches Economiques de Louvain 56, 383-390.

Pattanaik P.K., Xu Y., 2000. On Ranking Opportunity sets in economic environments. Journal of Economic Theory 93 (1), 48-71.

Sen A.K., 1988. Freedom of choice: Concept and Content. European Economic Review, 32, 269-294.

Sen A.K., 1991a. Welfare, preference and freedom. Journal of Econometrics 50, 15-29.

Sen A.K., 1991b. Markets and freedoms. Mimeo. 